\title{
Characterization of real and substitute birds through experimental and numerical analysis of momentum, average impact force and residual energy in bird strike on three rigid targets: a flat plate, a wedge and a splitter
}

Frederik Allaeys ${ }^{1}$, Geert Luyckx ${ }^{1}$, Wim Van Paepegem ${ }^{1}$ and Joris Degrieck ${ }^{1}$

${ }^{1}$ Department of Material Science and Engineering,

Ghent University, Technologiepark 903, 9052 Zwijnaarde, Belgium

Frederik.allaeys@UGent.be

\section{$\underline{\text { Abstract }}$}

To validate the increasingly used numerical models for optimization and verification of the designs subjected to bird strike, initial (calibration) tests are a necessity prior to full scale testing. Bird strike calibration tests on rigid targets specifically, give a valuable insight in the complex behaviour of a bird. This paper presents the results of a series of bird strike tests and simulations on three rigid targets (a plate, a wedge and a splitter) to quantify the forces originating from the change of momentum and splitting of the bird. In this study, momentum transfer is the key parameter to compare birds with different masses, materials, speeds, etc., as proposed in the reference works from the $20^{\text {th }}$ century. The main purpose of this paper is fourfold: (i) to introduce another way to measure momentum transfer on these kinds of structures and therefore get more consistent results, (ii) to show that gelatine generates similar impact forces as real birds, (iii) to point out that apart from the change of direction of the momentum, the deviatoric and/or dissipating constitutive behaviour of the bird also plays an important role and (iv) to show that a simple plate structure can be used to measure the residual energy of the bird remainders after an impact event. In a series of numerical simulations, the performance of a SPH bird with an EOS material model is used to validate the analytical models.

Keywords: Bird Strike, Momentum, Substitute Material, Force Measurement, Residual Energy

\section{Introduction}

Certification by analysis is a hot topic these days in bird strike robustness. But, a lot of research is still required to be able to prove that numerical methods are fully capable for simulating bird strike. An attempt to move in the right direction was taken in the European FP7 project called E-Break, where an important task is devoted to the development of a numerical model that is able to validate the design rules of the booster vane in terms of bird strike robustness and investigate the possibilities of Variable Stator Vane (VSV) systems. In order to achieve this, a series of calibration tests are performed to validate the numerical model before execution of the experiments on multiple sets of booster vanes. The purpose of this first study is to get an idea of the average impact forces and bird behaviour, and investigate the overall performance of numerical techniques. Rigid targets specifically, provide the opportunity to focus on the bird behaviour during impact.

The first bird strike tests on simplified structures date back to reference works from the $20^{\text {th }}$ century. There has always been a big focus on flat plate structures, rigid as well as deformable, since this kind of structure represents the most dangerous scenario for a bird strike and generates the highest 
impact forces. In this research, the influence of different impact speeds, masses and substitute materials can be investigated by comparing impact forces, strains, substitute bird behaviour, etc. Initial research on a rigid plate (orthogonally or rotated with respect to the impact direction) can be found in the well documented reports of Willbeck [1, 2], Barber et al. [3, 4] and Challita [5-7], where the effect of different porosities and substitute materials for the bird on the impact pressure and force was tested thoroughly. Allcock [8] already measured the impact force on different kinds of structures, such as a knife-like structure and a rigid nose. The reference works from the $20^{\text {th }}$ century showed that momentum transfer is the key parameter for comparison. Their results gave an idea of the main impact forces. More recent work on rigid plates exists as well [9]. Deformable flat plate experiments and simulations were performed on clamped aluminium square plates [10,11], on square prestressed composite plates clamped on two sides and simply supported on the other sides $[12,13]$ and clamped on one side [14-16] or circular composite plates clamped over their entire edge in [17].

Force is used as one of the main parameters to characterize these impact events. Force or momentum can be measured using multiple set-ups such as a ballistic pendulum [18], a Hopkinson bar [1, 3, 19], a spring in the form of calibrated beams [8], load cells [20-28], etc, all having their advantages and disadvantages. The actual shock behaviour and constitutive modelling of ballistic gelatine can be investigated using more fundamental set-ups such as the Hopkinson bar [29-31].

There is also a long history of development and testing of substitute materials that can fully represent the complex behaviour of a real bird [32]. There are multiple reasons to use a substitute material for the bird. No bird is the same, nor in size, weight or density, which on the other hand can be kept relatively constant when using a substitute material. Gelatine has become a generally accepted substitute material for the bird in experiments at high velocity (often with porosities), since the induced pressures are similar to a real bird and because it facilitates the reproducibility of the results [32].

Initial numerical research was performed on rigid flat plates to calculate pressure profiles, momentum and force using dedicated codes in [33] or by applying analytical bird loading models on the structure as in $[20,34,35]$. A majority of the more recent research on rigid flat plates focusses on the validation of the pressure at the centre of impact. Several papers also compare the deformation of the bird or the transferred momentum for different shapes, impact speeds and/or modelling techniques such as the standard Lagrangian, ALE, SPH or CEL method [36-42]. Numerically obtained momentum transfer for rigid rotated plates, wedges or splitter-like structures were not found in literature.

The main goal of this paper is to show the value of rigid target experiments and simulations characterized by the momentum transfer in bird strike research. This is done by revealing new insights that make it possible to evaluate the performance of substitute birds, focus on the differences between real and substitute bird behaviour and show the shortcoming of the state-ofthe-art numerical simulations. In this study, the momentum transfer shows to be the measure for the average impact force while being, in general, independent of the impact mass, velocity and shape. It will be used to analyse the impact on three different kinds of rigid target structures: a flat plate, a wedge and a splitter (Figure 1a). The idea is that a combination of the effects seen in these rigid 
target experiments (change of momentum direction, friction, splitting) will also be present in the experiments on the booster vanes (Figure 1b).

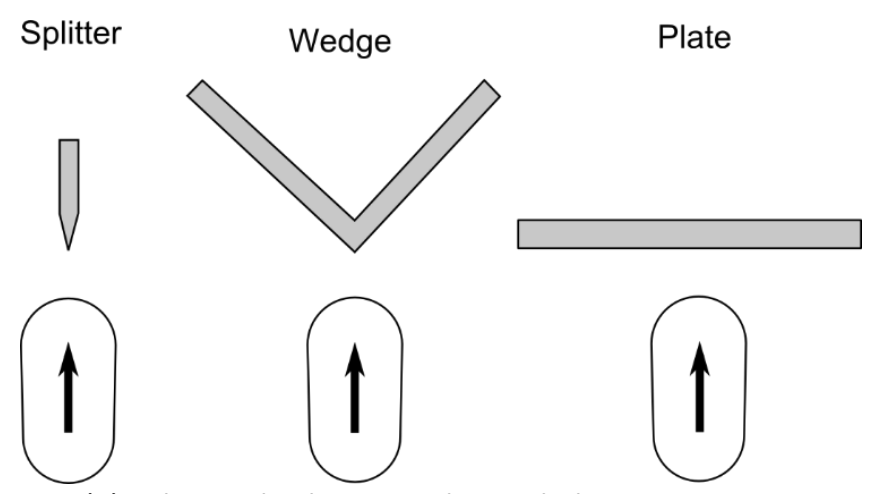

(a) The rigid splitter, wedge and plate structure.

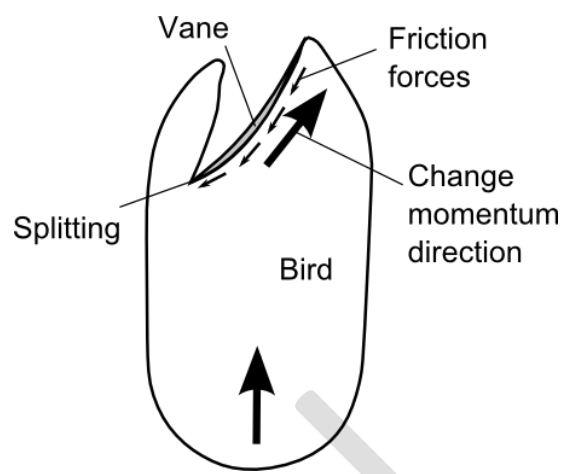

(b) Possible forces during bird strike on a booster vane.

Figure 1: Rigid target calibration tests and the possible forces.

Different gelatine to water ratios are tested (1:4, 1:6 and 1:9) as well as real birds with different masses and range of velocities to gain a better understanding of the main differences between gelatine and real birds. This will be done by using a new way to measure the transferred momentum in bird strike experiments. Finally, a new concept to measure the residual energy of a bird after an impact event with a plate structure will be introduced.

The next section will explain the preparation of the real and substitute birds, introduce the principle to measure the force and momentum transfer and the experimental set-ups. In section 3 , the momentum transfer for the three rigid targets will be examined analytically. After that, the numerical model is introduced briefly and the concept to measure the momentum transfer is validated. Section 5 will cover the results from the experiments and simulations. Finally, in section 6 , the concept for the residual energy measurement will be revealed, to end with a conclusion.

\section{Experimental set-ups}

In this section, first the gelatine substitute birds with different mixing ratios and the real birds (ducks and doves) used in this work are discussed. In section 2.2, the UGent bird strike test set-up is introduced and in section 2.3, the principle for the force measurement is explained and the three target structures are discussed.

\subsection{Birds}

For the experiments with gelatine, PBG05, mesh 20, from PB Gelatins is used [43]. Warm water around $40-60^{\circ} \mathrm{C}$ is mixed with the proper amount of gelatine and stirred for $10-20$ minutes. A small amount of coloured water paint is added to acquire high contrast between the plate and the originally translucent gelatine bird. With each bird preparation, a separate amount is made for density measurements at the time of the experiment. After moulding, the gelatine is cured in the fridge for 8-24 hours. Just before each experiment, the exact weight of the bird is determined by weighing the full and empty sabot. Three mixing ratios are tested: 1:4, 1:6 and 1:9, corresponding to a density of respectively $1060 \mathrm{~kg} / \mathrm{m}^{3}, 1040 \mathrm{~kg} / \mathrm{m}^{3}$ and $1030 \mathrm{~kg} / \mathrm{m}^{3}$. Bird masses of approximately 0.3 $\mathrm{kg}, 1.5 \mathrm{~kg}$ and $1.8 \mathrm{~kg}$ are shot at different speeds. The birds are cylindrically shaped with hemispherical ends and a length to diameter ratio of 2 (the length of the cylindrical part is therefore 
equal to the diameter of the hemispheres). For the $0.3 \mathrm{~kg}, 1.5 \mathrm{~kg}$ and $1.8 \mathrm{~kg}$ bird, this corresponds to a length of respectively $120 \mathrm{~mm}, 205 \mathrm{~mm}$ and $210 \mathrm{~mm}$ and a diameter of respectively $60 \mathrm{~mm}, 105$ $\mathrm{mm}$ and $115 \mathrm{~mm}$ (the $\mathrm{l} / \mathrm{d}$ ratio is not exact for the larger birds). There are two reasons to test the different gelatine mixing ratios. First of all, it is interesting to see what the influence is of the mixing ratio (MR) on the momentum transfer. Secondly, the initial 1:4 MR proved to behave very elastic in comparison with the birds.

Experiments with mallard ducks and doves are performed as well. For the experiments with mallard ducks, the birds are decapitated in advance. Keeping the neck as an extension of the body during launch was not feasible. Increasing the mass of the body by bending the neck and head and therefore adding extra mass to the body is not realistic as well, which is why the choice is made to decapitate the ducks. Wild mallard ducks are usually lighter than $1.5 \mathrm{~kg}$, as are the ones used in the four tests with ducks. Several doves are shot as well. The neck of the doves are not decapitated before launch as they can more easily be launched head first. The weight of the used doves is approximately 400 gram.

\subsection{The bird strike set-up}

The experiments are performed on the Ghent University bird strike set-up (Figure 2). The set-up is capable of shooting birds up to $42 \mathrm{~kJ}$. Birds can be launched with a weight of maximum $4 \mathrm{lb}$ (according to the regulations [44]) at speeds up to $250 \mathrm{~m} / \mathrm{s}$. At the beginning of each experiment, a projectile called a sabot is filled with foam in accordance to the desired shape, after which gelatine is moulded into the acquired foam shape. The sabot is mounted in front of a pressure vessel and released at the required pressure. After the release trigger, the sabot launches through a barrel and separates from the bird in the stripper chamber using a cone shaped stripper, after which the stripped bird flies into the test chamber and impacts on the required target. Before each experiment, the test chamber is evacuated up to 0.2 bar absolute pressure to be able to perform precise velocity measurements.
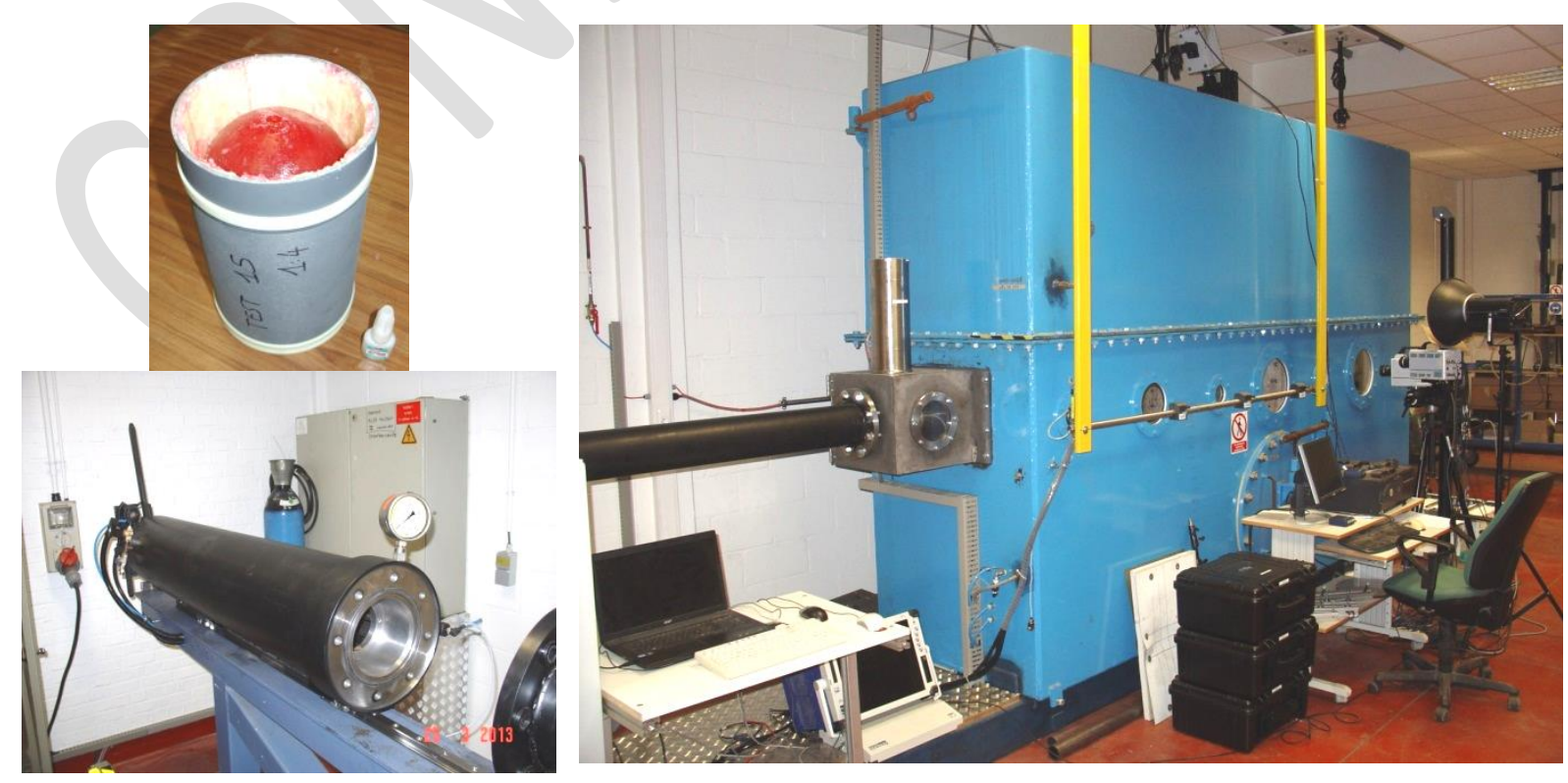

Figure 2: Ghent University bird strike test set-up. 


\subsection{Rigid targets}

In this section, first the new principle to measure the impact force is introduced. After that, the three rigid targets set-ups are discussed separately.

\subsubsection{Measurement principle for impact force and momentum transfer}

For the new principle to measure the force or momentum transfer in bird strike experiments, the three targets are mounted with one translational degree of freedom in the bird impact direction. By rewriting Newton's law and averaging the force integral, the average force can be obtained from the target velocity after impact:

$$
\begin{gathered}
F=m_{t} \cdot a_{t}=m_{t} \cdot \frac{d v_{t}}{d t} \\
\int m_{t} \cdot d v_{t}=\int F \cdot d t \\
m_{t} \cdot v_{t, 2}=F_{\text {avg }} \cdot \Delta t \\
F_{\text {avg }}=\frac{m_{t} \cdot v_{t, 2}}{\Delta t}=\frac{J_{t, 2}}{\Delta t}
\end{gathered}
$$

Where $t$ stands for target, $\Delta t$ is the total impact time, which can be approximated by the time that the bird needs to travel through its own length, or, be obtained directly from the high speed images if the shape deviates a lot from its original shape. $m_{t}$ and $v_{t, 2}$ respectively represent the mass and the final velocity of the target (the initial speed of the target $v_{t, 1}$ is 0 ) and $J_{t, 2}$ represents the target momentum $m_{t} \cdot v_{t, 2}$. This last equation shows that the average force $F_{\text {avg }}$ during impact is proportional to the momentum of the target after impact $\left(m_{t} \cdot v_{t, 2}\right)$. The momentum of the target is therefore a measure for the force. Equation 1 also implies that the force at a certain point in time is proportional to the derivative of the momentum of the target.

In this paper, the momentum of the target is normalized by dividing it by the impact momentum of the bird. The normalized momentum of the target can be seen as the amount of momentum that is transferred from the bird to the target structure or also, the momentum transfer (MT):

$$
M T=\frac{m_{t} \cdot v_{t}}{m_{b} \cdot v_{b, 1}} \cdot 100[\%]
$$

Where $m_{b}$ and $v_{b, 1}$ respectively represent the mass and the initial velocity of the bird. The momentum transfer can be considered at the end of the impact event $\left(v_{t}=v_{t, 2}\right)$ or in function of time $\left(v_{t}=v_{t}(t)\right)$. This expression is valid for each target and can be used to obtain the actual momentum transfer in the experiments and simulations.

In addition, for each target, a momentum balance can be made of the impact event, including the target and bird momentum and knowing that the target is at rest before impact. Because the direction of $v_{b, 2}$ does not necessarily coincide with the impact direction (e.g. for the rigid wedge), a switch to vector notation is made: 


$$
m_{b} \cdot \overrightarrow{v_{b, 1}}=m_{t} \cdot \overrightarrow{v_{t, 2}}+m_{b} \cdot \overrightarrow{v_{b, 2}}
$$

This equilibrium is the basis for the analytical models discussed in section 3. In combination with Equation 5 , the momentum balance is further elaborated in that section into an analytical expression for the momentum transfer.

Instead of determining forces directly via force transducers or a Hopkinson bar, the forces are derived from the measured velocities during impact. The weight of each set-up is chosen in such a way that the inertia limits the movement over which the target is accelerated during impact to a couple of millimetres. This first part of the velocity signal of each rigid target therefore consists of a very fast increase in velocity that lasts up to 1-2 ms (the actual impact). After the collision with the bird has finished, the rigid target continues to move over a distance of approximately $100 \mathrm{~mm}$ which results in a relatively constant velocity plateau. At the end, the rigid target is decelerated in a controlled way. Figure 3 shows an example of a plate velocity signal for a 300 gram gelatine bird impact experiment.

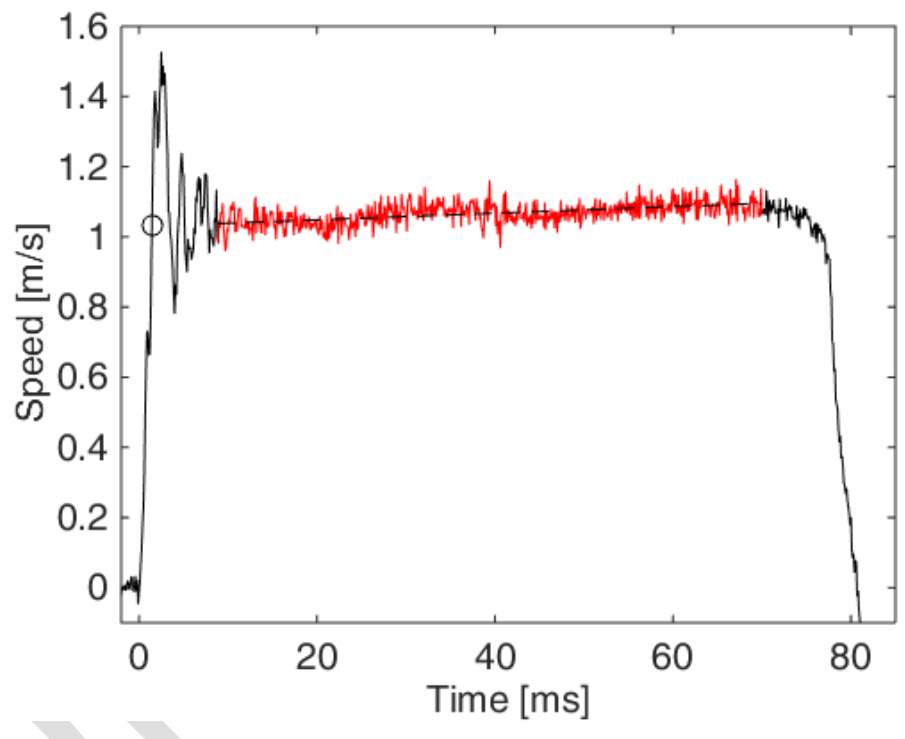

Figure 3: Velocity signal rigid plate.

Inevitably, oscillations are present in the velocity signal and an estimate of the obtained velocity of the target just after the collision needs to be made. For each experiment, a line is therefore fitted to the velocity plateau and evaluated at the first intersection with the velocity signal (the circle in Figure 3). This has the advantage of (theoretically) eliminating the influence of any friction in the bearings allowing the translational movement (a decrease of the velocity) and/or influence of the pressure wave behind the bird (which would increase the velocity).

By limiting the displacement during collision to a couple of millimetres, the impact of the bird is comparable to the impact onto a fixed target, but, the experiment is decoupled from the test chamber, minimizing the influence of the surroundings or boundary conditions on the experiment and guaranteeing safety. The set-up is also quite compact when compared to a Hopkinson bar or pendulum and can therefore easily be used inside the vacuum chamber. 


\subsubsection{Plate}

The rigid plate set-up (Figure 4) consists of a stiff $400 \times 600 \mathrm{~mm}^{2}$ steel plate reinforced sandwich panel bolted to a stiffened steel frame (not visible on the images) and attached to a linearly movable box (for smaller birds, instead of the sandwich panel, a $12 \mathrm{~mm}$ Al plate is bolted to the stiff steel frame). The linear movement of the box is realised using four needle bearings. The friction induced by these bearings is negligible compared to the high inertia forces acting in this type of experiment.
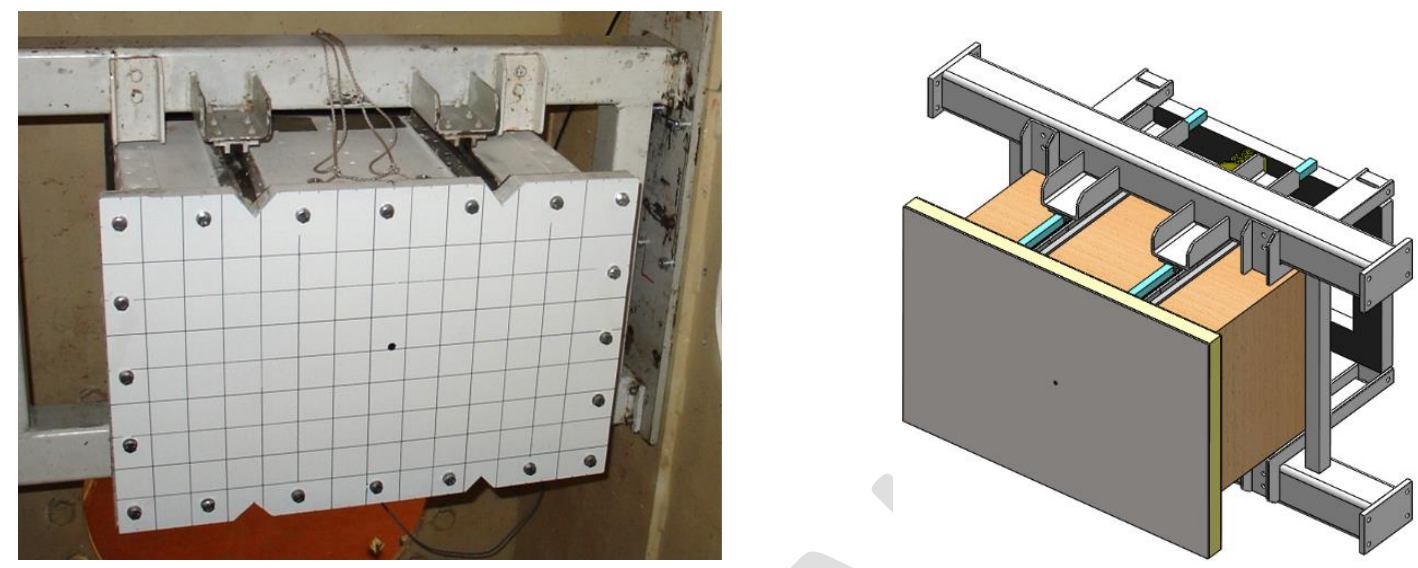

Figure 4: Plate set-up.

At the back, steel crushing tubes or honeycomb cardboard is used to decelerate the box over a distance of approximately $50 \mathrm{~mm}$.

\subsubsection{Wedge}

The wedge consists of a reinforced steel L-profile that fixes two aluminium plates orthogonally with respect to each other (Figure 5). The plates have a height of $300 \mathrm{~mm}$ and a length of $350 \mathrm{~mm}$. It is designed in such a way that the inertia (and stiffness) is located at the centre of impact to minimize oscillations. The initial idea was to use a rotated plate. But, a wedge has the advantage to have, in theory, no reaction force in the direction perpendicular to the impact direction. The linear translation is again guaranteed by four linear needle bearings. The weight of the wedge is $21.7 \mathrm{~kg}$.

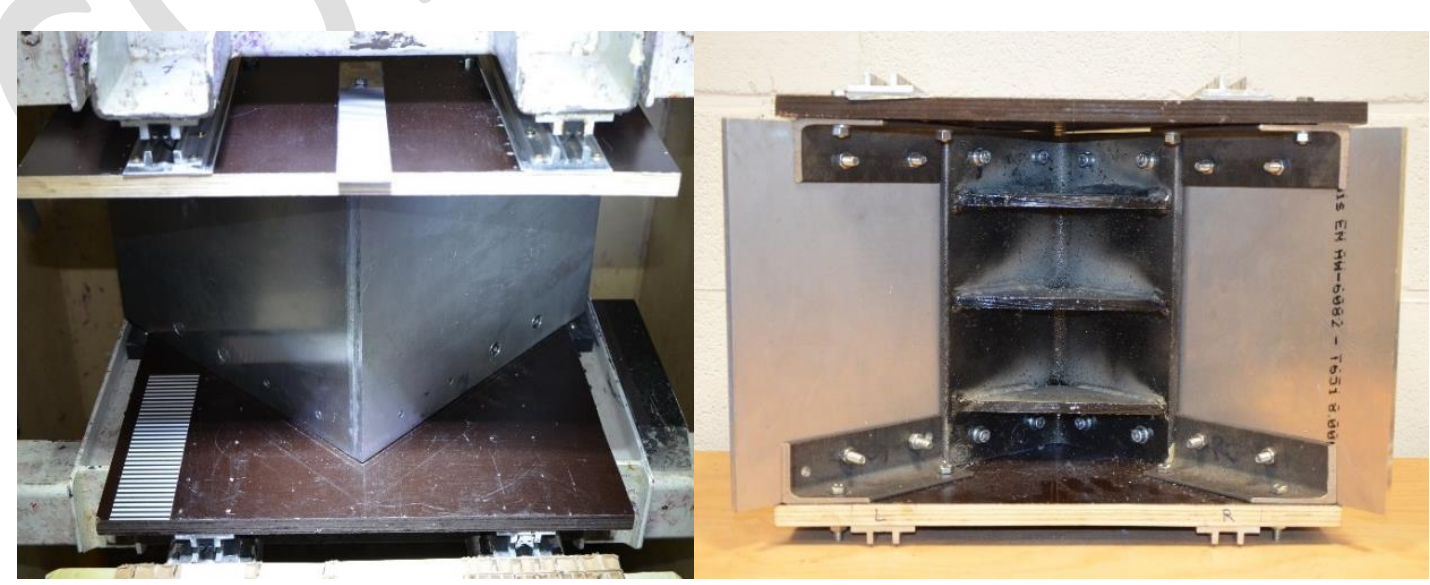

Figure 5: Wedge set-up. 


\subsubsection{Splitter}

The splitter is a $12 \mathrm{~mm}$ thin knife-like structure with a height of $200 \mathrm{~mm}$ (Figure 6). It consists of a foam core with steel plates at each side. The actual tip of the splitter is a machined, almost entirely solid piece with a fillet of approximately $1 \mathrm{~mm}$ and a tip angle of $18^{\circ}$. The linear movement is ensured using needle bearings in $\mathrm{V}$ shape (one at the bottom and one on the top). The final weight of the splitter including sensors is $1.73 \mathrm{~kg}$.

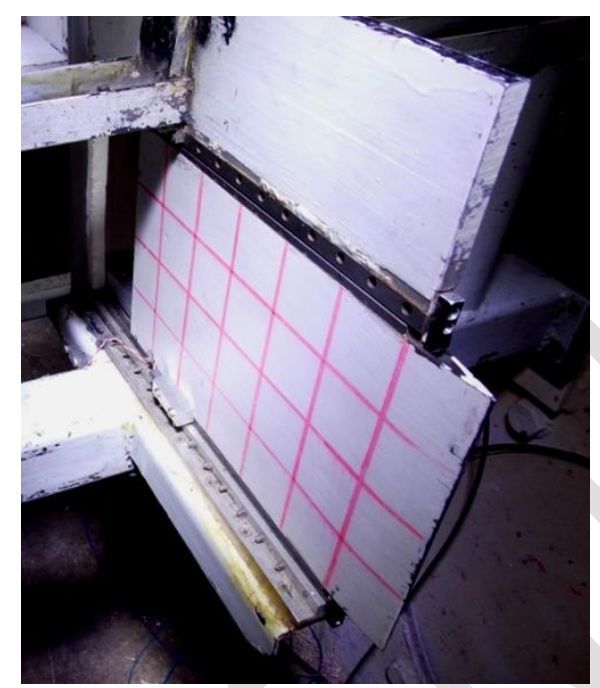

Figure 6: Splitter set-up.

For the splitter and the plate, a Kübler Limes LI50/B2 linear displacement transducer and at least one PCB 350B24 (5000g) accelerometer are available. These sensors are sampled with a HBM Gen5i oscilloscope (1 $\mathrm{MHz}$ for the accelerometer and $10 \mathrm{MHz}$ for the displacement transducer). One APX-RS and two SA-4 Photron high speed cameras are used to record the experiments from different angles. For some experiments, optical tracking of the displacement of the target is also done using these high speed cameras. For the Kübler Limes and optical tracking measurements, numerical differentiation of the displacement signals is performed to obtain the corresponding velocity signals and for the accelerometer measurements, the accelerations are integrated.

In the next section the analytical models will be introduced.

3. Analytical models - force and momentum transfer

3.1 Plate

For the plate, the experiments showed that the velocity of the bird in the impact direction is zero after impact (the bird material moves only in the radial direction). Considering the conservation of momentum (Equation 6 ) in the impact direction and equating $v_{b, 2}$ to 0 , it can be deduced that the momentum of the plate is equal to the impact momentum of the bird. As a result, the momentum transfer after impact is $100 \%$ (Equation 5) or also, the momentum of the bird is entirely transferred to the plate.

According to the analytical model, the momentum transfer is always $100 \%$, independent of the shape, the material, mass or velocity of the impactor. This reveals the advantage of momentum transfer over average force. Equation 4 contains $\Delta t$, which depends on the velocity and the length of 
the bird. The length of a real bird is in general slightly larger and for the substitute birds, the volume does not scale linearly with the length of the bird (the volume does not have a linear relationship with the length because of the fixed length to diameter ratio). The mass thus also has a non-linear influence on the force. To compare the different impactors, with different masses and velocities, the momentum transfer therefore has a considerable advantage over the force.

In the plate experiments, large oscillations were present in the plate velocity curves after impact, indicating that the target structure was not rigid enough. The force at each time step therefore will not be represented by the current change in velocity of the rigid target (Equation 1). However, simulations showed that elastic deformation of the target structure has a minor influence on the final momentum transfer.

\subsection{Wedge and splitter}

Figure 7 gives several schematic overviews that will clarify the momentum balance for the wedge (the same principle can be applied to the splitter). Because there is a fundamental difference with a rotated plate, this will be covered first (first picture in Figure 7). It can be assumed that after impact, there is no momentum of the bird perpendicular to the plate (the $y$-direction). To avoid the speed of the bird after impact $v_{b, 2}$ in the momentum balance, Equation 6 is first considered in the $y$-direction. The initial momentum of the bird in the $y$-direction is indicated by the green vector. This green vector is equal to the initial momentum times the sinus of the angle $\alpha$ in which the bird is deflected. From the momentum balance, the target momentum is obtained:

$$
J_{t, 2}=m_{b} \cdot v_{b, 1} \sin (\alpha)
$$

When the momentum is measured in the impact direction however, only a projection of $J_{t, 2}$ is measured, indicated by the upper triangle. The target momentum in Equation 7 therefore has to be projected again on the impact direction (by multiplying it again with $\sin (\alpha)$ ). To obtain the momentum transfer, it is also divided by the bird impact momentum:

$$
M T_{\text {impact direction }}=\sin (\alpha)^{2} .100[\%]
$$

Together with Equation 5, it can be said that the momentum transfer is defined by $\sin (\alpha)^{2}$. For an angle of $45^{\circ}$, the momentum transfer would be $50 \%$ of the initial momentum. Experiments on rotated rigid plates with porous gelatine and real birds, for angles of $90^{\circ}, 45^{\circ}$ and $25^{\circ}$ have been investigated by Wilbeck and Barber [1,3]. If friction forces would have been present, the momentum transfer would have increased by a certain amount above the theoretical momentum (because a high normal force would be generated, which would result in a friction force that has a considerable component in the measurement direction). The momentum transfer however was as predicted by the analytical model without friction (within the obtained scatter). Allcock and Collin used a similar reasoning to obtain the momentum transfer for wedge like structures [8]. They obtained the momentum transfer by mistakenly considering two times the impact of half a bird on a rotated plate to obtain the same momentum transfer, as shown in the middle figure 7. This is not correct. Considering the two halves of a bird separately requires the use of a symmetry force, as indicated in the third figure. If only one half of the bird would impact the wedge and there would be another 
plate in the symmetry plane, a pressure would be exerted on that plate which proves the necessity of a symmetry force. Symmetry implies that shear forces cannot be present at the symmetry plane, which is why the symmetry force can only be horizontal.
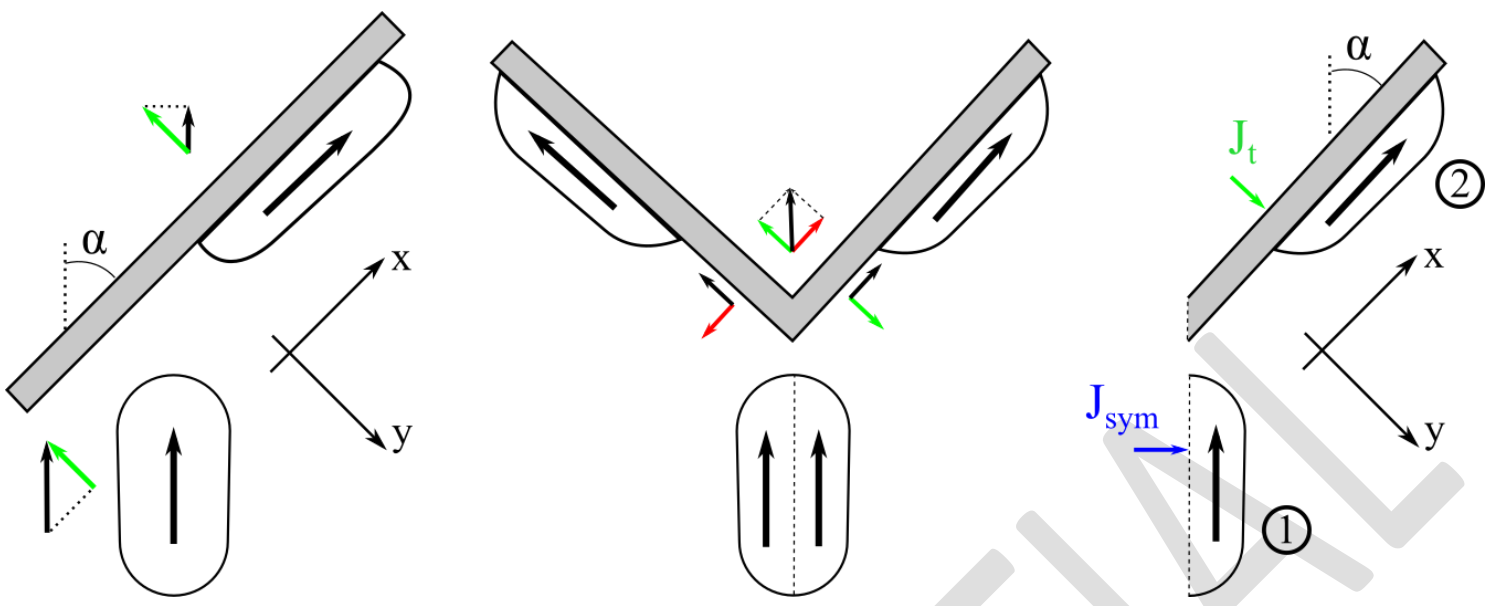

Figure 7: Schematic representations of the impact momentum for the wedge.

The momentum balances in both the $\mathrm{x}$ - and $\mathrm{y}$-direction are now necessary to obtain the momentum transfer:

$$
\begin{gathered}
x: \quad J_{\text {sym }} \sin (\alpha)+\frac{m_{b} v_{b, 1}}{2} \cos (\alpha)=\frac{m_{b} v_{b, 2}}{2} \\
y: J_{t, 2}+J_{\text {sym }} \cos (\alpha)=\frac{m_{b} v_{b, 1}}{2} \sin (\alpha)
\end{gathered}
$$

Where $J_{\text {sym }}$ is the momentum equivalent to the symmetry force defined earlier, $J_{t, 2}$ is the target momentum equivalent to the reaction force of the plate, $v_{b, 1}$ and $v_{b, 2}$ is respectively the velocity of the bird before and after impact and $\alpha$ is the angle over which the bird deviates from its initial direction. Combining these two equations by eliminating $J_{\text {sym }}$, equating for $J_{t}$, projecting the momentum on the impact direction, doubling the result to account for the other half and dividing by the initial momentum, results in the following expression for the transferred momentum:

$$
M T=\left(1-\frac{v_{b, 2} \cos (\alpha)}{v_{b, 1}}\right) \cdot 100[\%]
$$

This expression can be used for the wedge and also for the splitter. Obtaining the analytical solution in the experiments for the wedge requires the speed of the bird after impact $v_{b, 2}$, which cannot be determined accurately from the experimental data. In the simulations on the other hand, $v_{b, 2}$ can be determined, as will be shown in the next section. Since Equation 5 (which can be derived from experimental measurements and simulations) and Equation 11 (derived from simulations) both calculate the same momentum transfer, they can be used interchangeably and can validate one another. Further, if the bird in the experiments remains quite intact after impact and its velocity $v_{b, 2}$ can be estimated from high speed images, the momentum transfer can be measured in two independent ways (through target velocity and bird velocity). 
For the splitter, the assumption can be made that a negligible amount of kinetic energy is dissipated in the bird $\left(v_{b, 2}=v_{b, 1}\right)$, which results in a theoretical momentum transfer of $1.23 \%$ for a deviation angle $\alpha$ of $9^{\circ}$ (the tip angle of the stripper is $18^{\circ}$ ).

For each target, the analytical expressions derived in this section will be compared to the actual transferred momentum in the experiments and simulations derived from the momentum of the target (Equation 5).

In the next section, first the numerical model is introduced and the principle to measure the force is validated.

\section{4. $\quad$ Numerical model}

Explicit simulations are executed with $\mathrm{Abaqus}^{\mathrm{TM}}$ to validate the analytical models. In these simulations, the bird is modelled with smoothed particle hydrodynamics or SPH. SPH is increasingly used in bird strike simulations as it already proved to be quite capable of simulating high deforming matter with defragmentation [32]. A complete and clear explanation of SPH and its governing equations can be found in literature [45].

Structured meshes are used for all the bird meshes. A characteristic mesh size of $2 \mathrm{~mm}$ proved to be sufficiently converged for most targets. For the bird material model, a linear Mie-Gruneïsen EOS is used, which relates the pressure to the density. Parameters for porcine gelatine can be found in literature $(\mathrm{c} 0=1570 \mathrm{~m} / \mathrm{s}$ and $\mathrm{s}=1.77)$ [30], which is very similar to water. To reduce the high artificial dissipation, the linear and quadratic bulk viscosity factor are scaled down to $20 \%$. A tensile failure criterion of $1 \mathrm{MPa}$ is set as well.

The targets are modelled as rigid objects (using analytical surfaces or solids with rigid constraints). Each target is fixed to a reference node able to move in the direction of impact. Extra inertia is added to achieve a similar mass as the set-ups. To verify that the translation DOF has a negligible influence on the impact, a comparison with a fixed boundary condition is made for the plate target. The reaction force of the plate in the direction of impact is integrated over time, giving the momentum of the fixed boundary condition (Equation 2). The momentum of the plate with the translation DOF is obtained by multiplying the mass of the target with the velocity of the target $\left(m_{t} \cdot v_{t}(t)\right)$. This is done for a 300 gram bird with a mesh size of $2 \mathrm{~mm}$. The result is depicted in Figure 8. The momentum curves lie on top of each other, validating the concept to measure the momentum or force. 


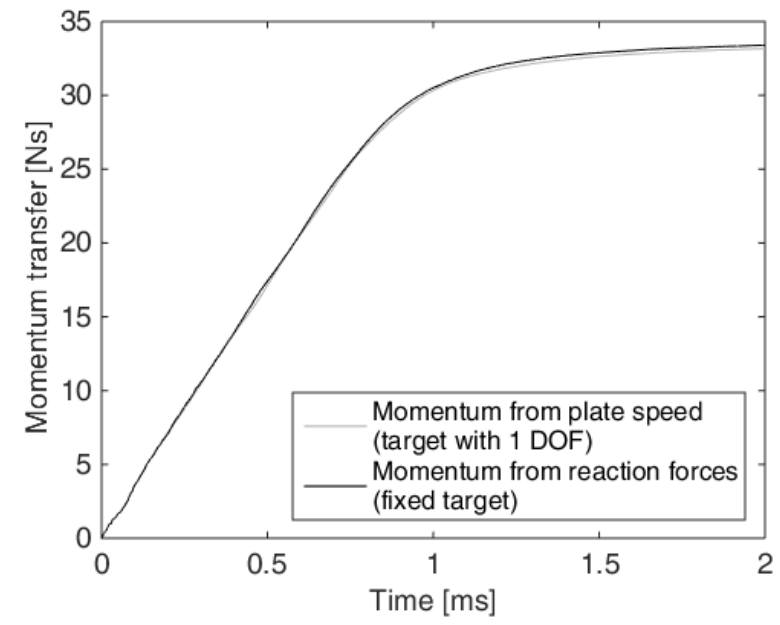

Figure 8: Momentum of the plate structure obtained from the plate speed and from the reaction forces.

To obtain the analytical momentum transfer for wedge-like structures, the speed of the bird after impact $v_{b, 2}$ needs to be known (Equation 11). It is impossible to acquire this accurately from the experimental data. For the simulations on the other hand, a script is written that calculates the momentum from the velocity vector of each separate particle. Because SPH is a Lagrangian technique, there is no mass diffusion between the elements and the material stays fixed with respect to the particle motion. Structured meshes are used in this work, the particle mass therefore can be calculated directly from the density and the characteristic volume corresponding to the mesh size.

In the next section, the results from the experiments and simulations are discussed.

\section{Results of the experiments and simulations}

Figure 9 shows the momentum transfer for the three rigid targets in function of impact speed (impact speed instead of impact momentum to get a better overview of the results). All data points are derived from the measurement of the target velocity after bird collision and calculation of Equation 5. All the birds are shot at a velocity between $60-150 \mathrm{~m} / \mathrm{s}$. To get an idea of the absolute values of the average impact forces, a force graph is given as well (Figure 10). How representative the average force is for the instantaneous force over time will be covered further on in this section. 


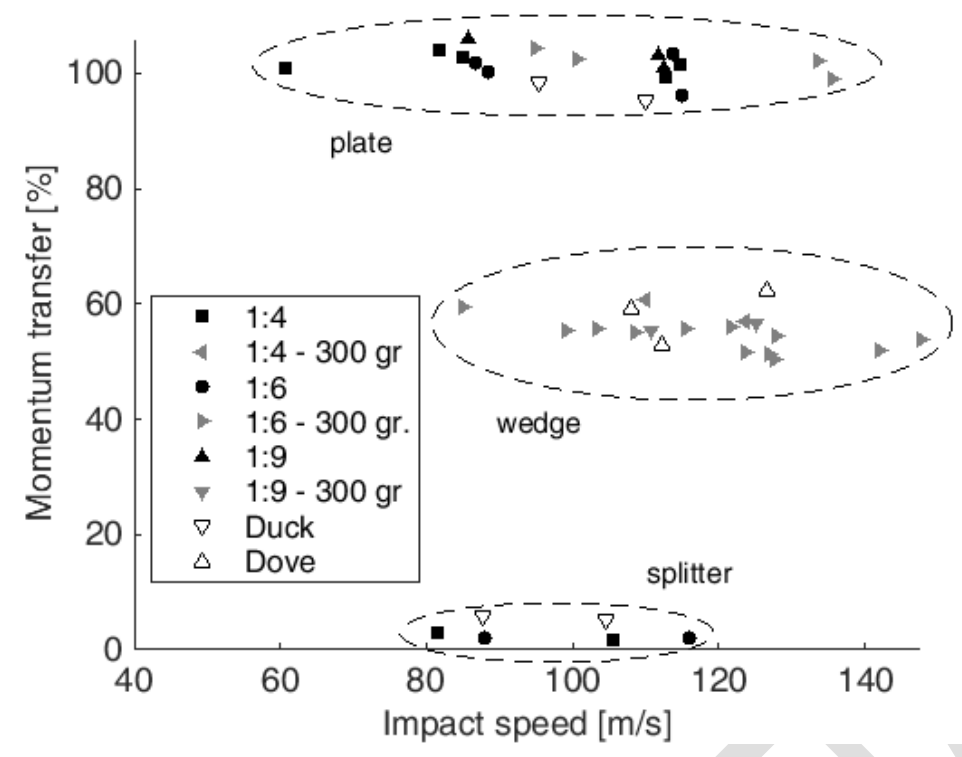

Figure 9: Momentum transfer in the plate, wedge and splitter experiments.

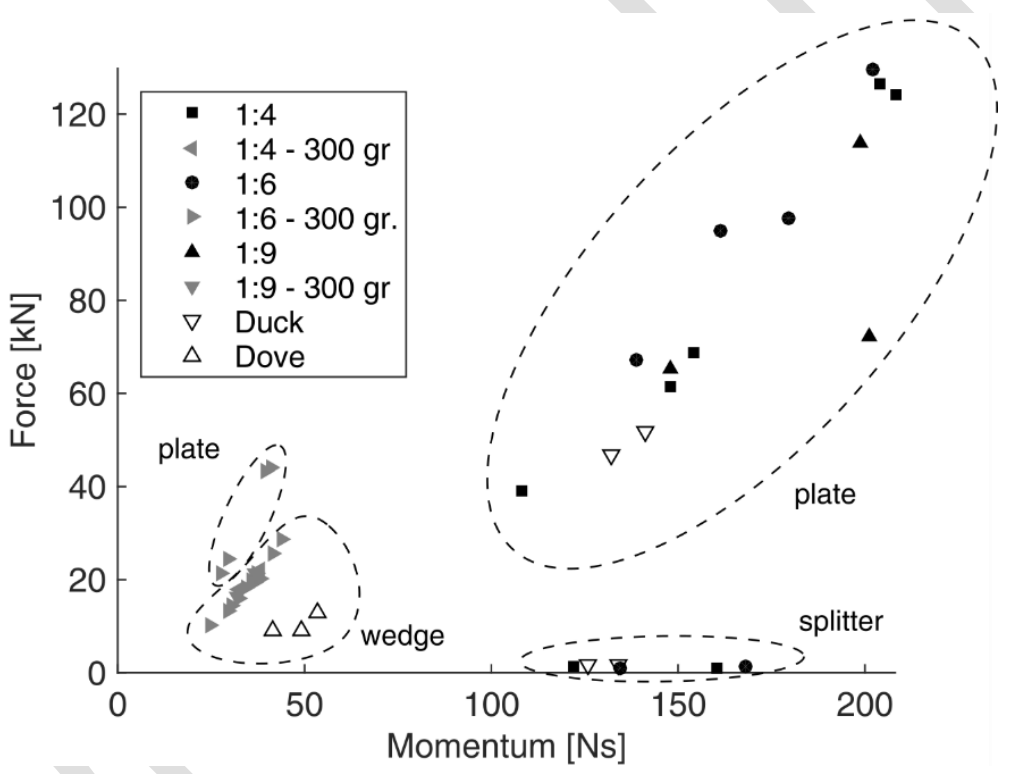

Figure 10: Average impact force in the plate, wedge and splitter experiments.

To give a better perspective on the consistency of the results, a graph relating the output momentum with the input momentum for the rigid plate experiments is shown in Figure 11, as was also done in the reference work of Wilbeck [1]. The $100 \%$ momentum transfer expected from the analytical model translates to a one-to-one relation between input and output momentum, which can be observed in the graph. 


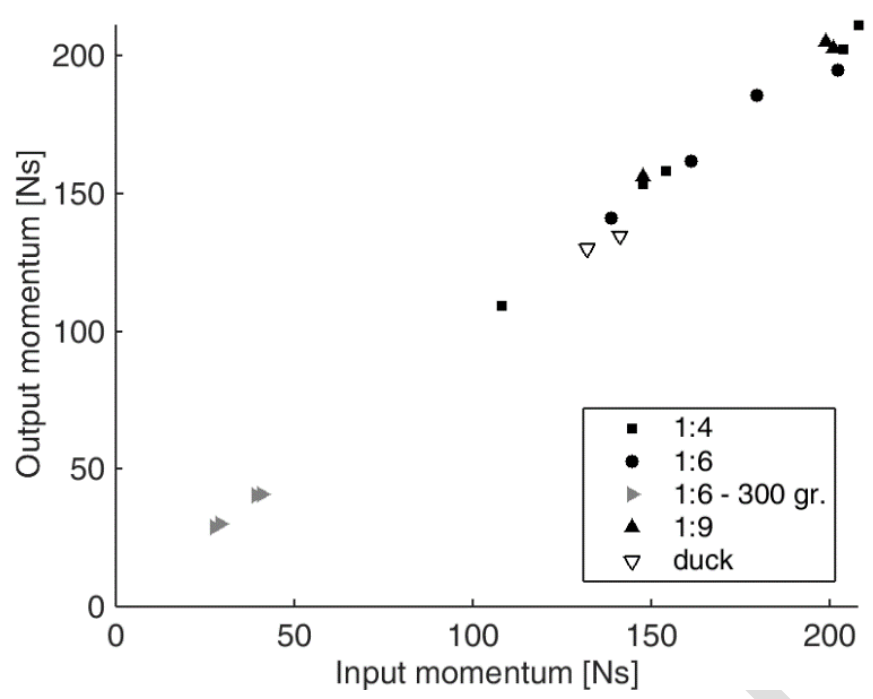

Figure 11: Output momentum versus input momentum for the plate experiments.

Still, some scatter can be observed in the rigid plate and wedge experiments. Multiple error sources can be identified: the measurement of the bird velocity, the measurement of the target velocity, pressure waves accompanying the bird, and also debris that enters the test chamber. During the stripping process, the bird is stripped from the sabot through the supporting foam. Inevitably, some foam enters the chamber. An investigation of several lumps of foam indicates that the amount of foam can increase the momentum transfer up to $1-2 \%$. On the other hand, a small misalignment due to the crushing of the supporting foam in the sabot might cut off a slice of the bird during impact.

In the following subsections, the results for the three targets will be examined separately.

\subsection{The plate}

\subsubsection{Experiments}

The momentum transfer for the plate target is close to $100 \%$, as predicted by the analytical model. There is no clear influence of the bird material on the order of magnitude of the observed scatter seen in the results. A mean value of $101.2 \%$ momentum transfer is obtained from the experiments. The slightly higher momentum transfer can be the result of lightweight foam that encloses the bird. The figure below (Figure 12) shows an example of a $1.6 \mathrm{~kg}$ 1:6 MR gelatine bird impacting the plate structure at $84 \mathrm{~m} / \mathrm{s}$. Some foam can be observed.

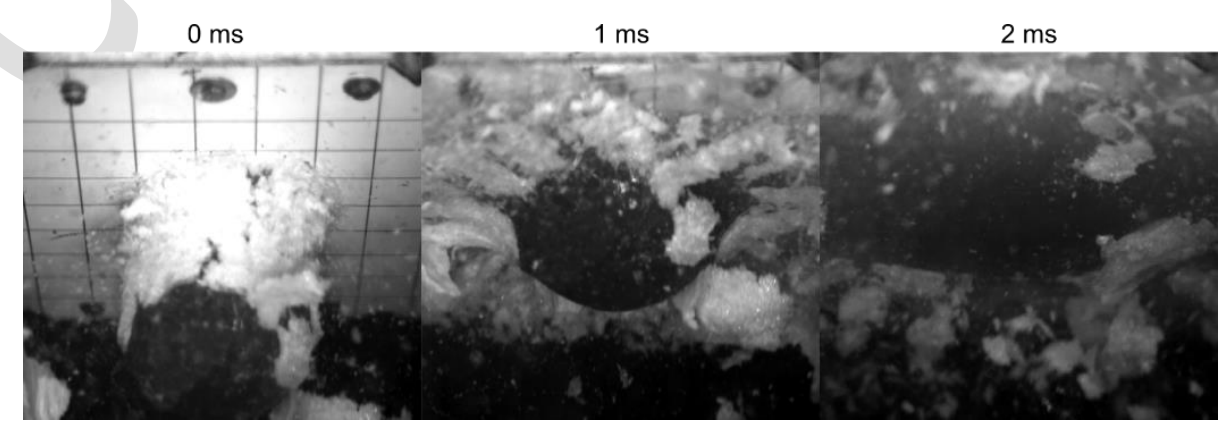

Figure 12: Impact of a $1.6 \mathrm{~kg}$ 1:6 MR gelatine bird on the rigid plate target. 
The consistent $100 \%$ momentum transfer is an interesting observation. To get a momentum transfer similar to a real bird, the substitute material should be able to disintegrate in the radial direction at the observed velocities, with zero momentum in the impact direction after impact. Gelatine clearly behaves according to this assumption.

Linear trends can be observed in the force plot (Figure 10). For the 300 gram birds, the force increases faster than for the bigger birds. As mentioned before, this is because the impact time (related to the length of the bird) does not decrease as fast as the mass for smaller birds (when using a fixed $\mathrm{I} / \mathrm{d}$ ratio, see also Equation 4). Some outliers can be found. In the experiment at approximately $200 \mathrm{Ns}$ and $70 \mathrm{kN}$, a 1:9 gelatine mixture was used. Because of the low strength of the 1:9 mixture birds, it is more difficult to keep the bird in its original shape throughout the launching process. In this specific case, the bird was stretched out quite a lot, resulting in a significantly higher impact time and therefore lower average force. The momentum transfer and force for the ducks are also slightly lower. Compared to the experiments with gelatine birds, no foam is present which could be an indication that some mass is cut off during the stripping process. The lower force is also partly the result of the fact that the ducks are roughly $150 \%$ longer than the gelatine birds.

Decreasing the gelatine MR (decreasing the amount of gelatine in the mixture) decreases the size of the gelatine lumps after impact. The 1:9 MR bird deformed in fluid-like manner and almost completely disintegrated after impact (Figure 13), which is not comparable to the duck. The shape is also very hard to maintain during acceleration and stripping of the bird, which is why a 1:9 MR bird is not ideal as substitute bird in a booster vane experiment. The 1:4 and 1:6 MR birds resulted in big lumps after impact. A 1:6 MR naturally resulted in smaller lumps, more comparable to the ducks. The big lumps after impact are a first indication that gelatine is capable of storing a high amount of elastic energy.

$1: 4$

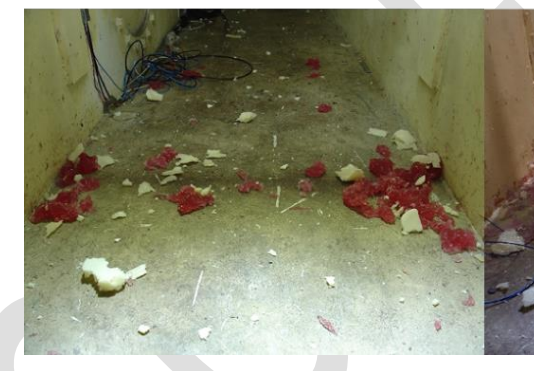

$1: 6$

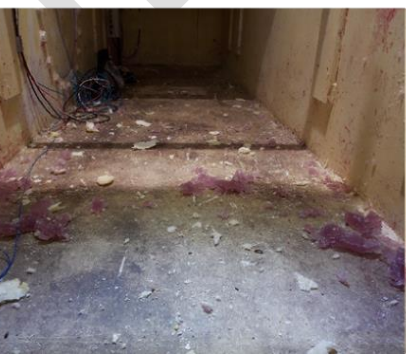

$1: 9$

Figure 13: Influence of the gelatine MR on the lumps after impact.

\subsubsection{Simulations}

Inherent to bird strike experiments, small deviations can be found in the impact conditions: the bird can deviate slightly from the original shape, the bird might rotate during flight, resulting in a certain impact tilt angle, etc. Several simulations with a range of aspect ratios ( $1 / \mathrm{d}=1,1.5,2,3$ and 4$)$, tilt angles $\left(0^{\circ}, 10^{\circ}, 15^{\circ}, 30^{\circ}\right.$ and $\left.45^{\circ}\right)$ and bird masses ( $\mathrm{m}=0.11 \mathrm{~kg}, 0.17 \mathrm{~kg}, 0.30 \mathrm{~kg}, 0.42 \mathrm{~kg}$ and $0.79 \mathrm{~kg}$ ) are performed to further investigate the influence of such deviations. The aspect ratio and tilt angle simulations are performed with a 300 gram bird with a mesh size of $2 \mathrm{~mm}$ containing roughly 36.000 elements. For each simulation, the momentum transfer in function of time is shown in Figure 14. 

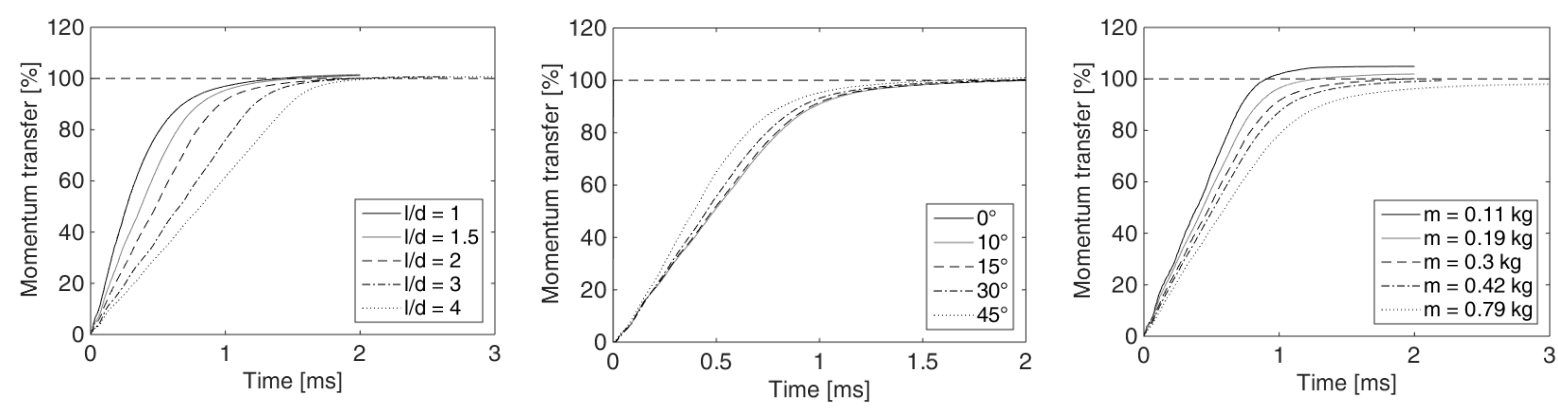

Figure 14: Influence of aspect ratio (left), tilt angle (middle) and bird mass (right) on the momentum transfer in the simulations.

These momentum curves again indicate that the momentum transfer is independent of the shape. For the $0.11 \mathrm{~kg}$ bird, the momentum transfer is slightly higher. This is a result of the reduced amount of particles that represent the bird. A finer mesh is needed to obtain a converged solution for the smaller bird. The slope of the curves before reaching $100 \%$ is a measure for the force. The aspect ratio clearly has a big influence on the slope or force, the tilt angle not so much and as already mentioned before, the average impact force relative to the impact momentum increases when the mass is decreased.

\subsection{Wedge}

\subsubsection{Experiments}

For the three considered mixing ratios, as well as for the doves, a momentum transfer between 50 $60 \%$ is obtained. According to the analytical model, this would mean that the velocity after impact is always lower than the impact velocity and that the velocity after impact relative to the impact speed is comparable for all the impactors.

Figure 15 shows a $0.3 \mathrm{~kg} 1: 6 \mathrm{MR}$ gelatine bird impact at $100 \mathrm{~m} / \mathrm{s}$.

$0 \mathrm{~ms}$

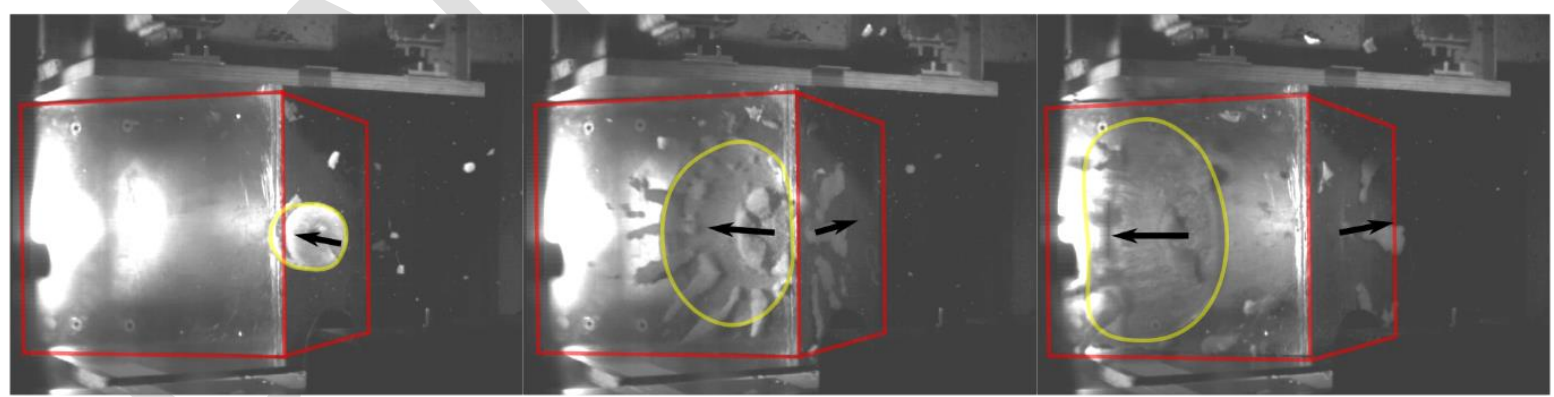

Figure 15: Impact of a $0.3 \mathrm{~kg}$ 1:6 MR gelatine bird (yellow contour for left part) on the wedge (red contour).

What is striking, is the amount of elastic deformation of the gelatine, even at these high impact velocities. This can also be observed in the momentum transfer graphs. Figure 16a shows the momentum transfer over time for three experiments with different gelatine mixing ratios and two experiments with doves. A 300 gram gelatine bird travels through its own length in approximately 1 $\mathrm{ms}$, the momentum however is transferred over a time span which is rather in the order of magnitude of $2 \mathrm{~ms}$ (i.e. the momentum transfer keeps increasing up to $2 \mathrm{~ms}$ after the start of 
impact), which can only be the result of kinetic energy that is temporally transferred to elastic energy during impact. The momentum transfer of the real birds takes even longer. This is partly the result of the length of the bird. In the experiments with the doves, the neck is stretched out in the impact direction. The head and neck therefore impact approximately $1 \mathrm{~ms}$ earlier than the body. As a result, the average impact force for the doves in Figure 10 is also considerably lower than for the gelatine birds. Shifting the curves $1 \mathrm{~ms}$ to the left (Figure 16b) shows that the slope of the curves, which is a measure for the force, is quite similar for the doves and the gelatine birds. Also, the momentum transfer graphs of the gelatine birds and the doves are all between 50 and $60 \%$. Regardless of the head and the neck, gelatine is therefore able to capture the average force and momentum transfer of a dove quite well.

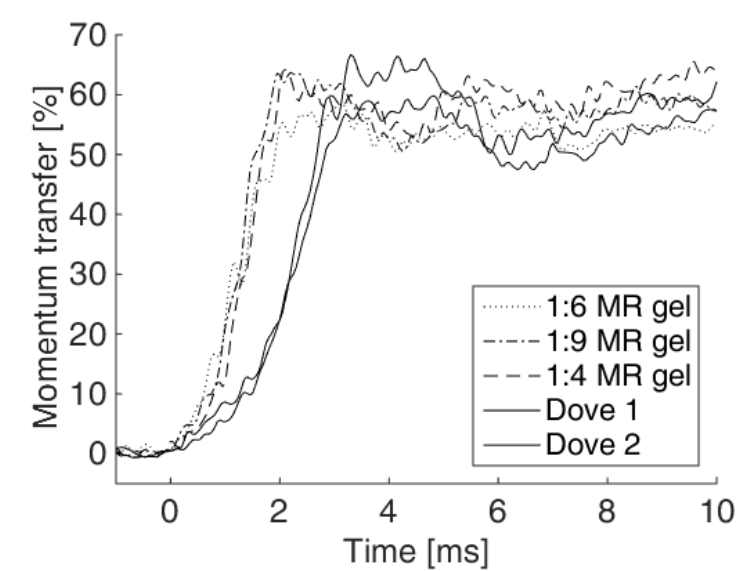

(a) synchronized at the start of impact

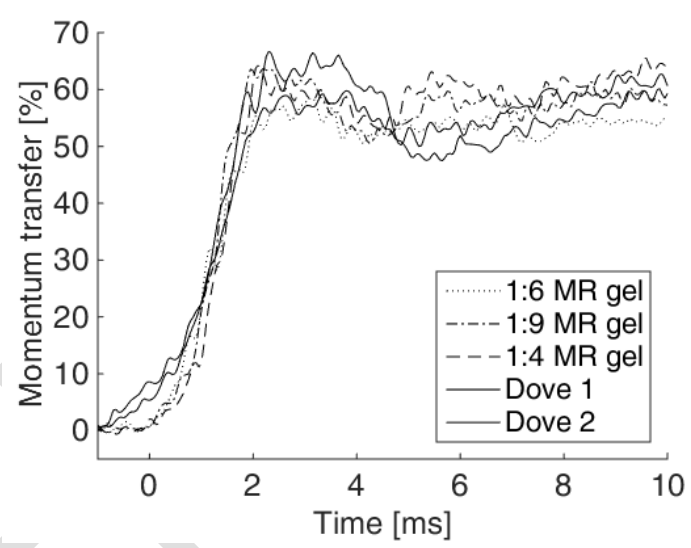

(b) shifted the dove momentum curves over the impact time of the head and neck of the doves

Figure 16: Momentum transfer curves for wedge experiments with different impactors (three different gelatine mixing ratios and two doves).

\subsubsection{Simulations}

The analytical models introduced in section 3.2 showed that contrary to the wedge, the momentum transfer of a rotated plate is independent of the speed of the bird after impact. To validate this and consequently reveal the significant difference between the rotated plate and the wedge, simulations are performed both on a rotated plate and on a wedge structure. Figure 17a shows the final momentum transfer of the rotated plate for an angle of $\alpha=30^{\circ}, 40^{\circ}, 45^{\circ}, 60^{\circ}$ and $70^{\circ}$, compared to the analytical solution (Equation 8). The momentum transfer correlates well with the analytical solution.

Next Figure 17b shows the simulated momentum transfer over time of the wedge $\left(\alpha=45^{\circ}\right)$ for different bird mesh sizes $(8,4,2$ and $1 \mathrm{~mm})$, based on the target velocity and Equation 5 . The momentum transfer for the wedge converges to a value of about $37 \%$. To validate the analytical expression of the momentum transfer for the wedge (Equation 11), the speed of the bird after impact $\mathrm{v}_{\mathrm{b}, 2}$ can be derived using the script mentioned in section 4 . For the $2 \mathrm{~mm}$ bird mesh in Figure $17 \mathrm{~b}, \mathrm{v}_{\mathrm{b}, 2}$ is calculated as $115.6 \mathrm{~m} / \mathrm{s}$ for an initial bird impact speed $\mathrm{v}_{\mathrm{b}, 1}=130 \mathrm{~m} / \mathrm{s}$, which translates to a momentum transfer of $37.1 \%$ (Equation 11 ). This is very close to the momentum transfer obtained from the velocity of the plate in Figure 17b. This proves that the momentum balance is valid. 
Despite the fact that the momentum balance is correct (both analytically and in simulation), the momentum transfer in Figure $17 \mathrm{~b}$ is much lower than the $50-60 \%$ seen in the experiments (see also Figure 9). The fact that the momentum transfer in the experiments is much higher can only be because of a lower $v_{b, 2}$ (since Equation 11 has been proved to be valid). The momentum is also transferred over a much shorter period in the simulations (the momentum transfer keeps rising up to roughly $1 \mathrm{~ms}$, opposed to the $2 \mathrm{~ms}$ in the experiments, Figure 16a). These two observations give an indication that the material model is not capable to simulate this event (the momentum transfer is underestimated by $20 \%$ ). Some deviatoric and/or dissipating constitutive behaviour could decrease the speed after impact $v_{b, 2}$ and therefore increase the momentum transfer. Deviatoric stiffness for sure is missing to increase the impact time and obtain a more elastic behaviour.

This type of target hereby proves to be very valuable when correlating bird strike simulations with experiments as the momentum transfer depends greatly on the constitutive behaviour of the impactor material. The wedge target can be used to test new material models in the future.

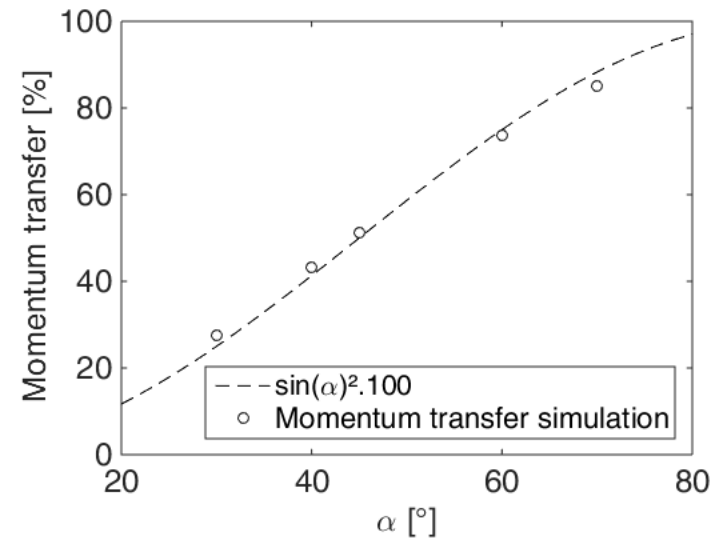

(a) Final momentum transfer for the rotated plate, in function of the angle with the impact direction.

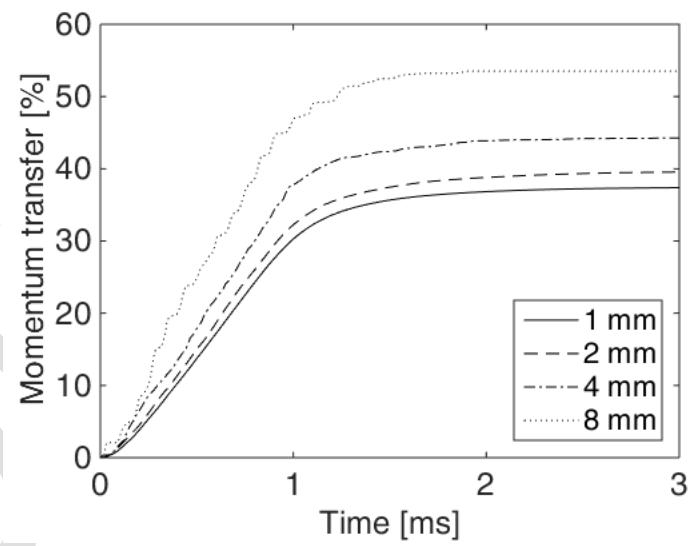

(b) Wedge momentum over time for different bird mesh sizes.

Figure 17: Momentum transfer for the rotated plate and the wedge.

\subsection{Splitter}

\subsubsection{Experiments}

A zoom on the momentum transfer and force results for the splitter is shown in Figure 18.

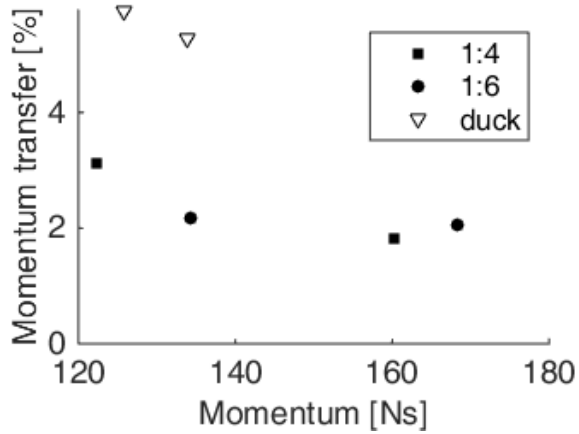

(a) Momentum transfer

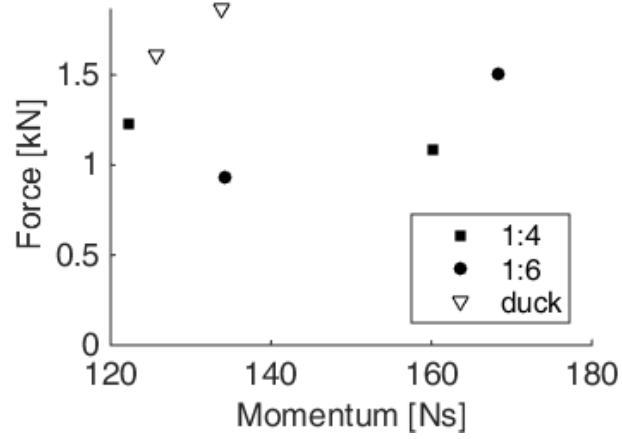

(b) Average impact force 
Figure 18: Momentum transfer and force results of the splitter experiments.

The momentum transfer for the gelatine birds is between 1.8-3.1\% for both mixing ratios. These values can be compared to the analytical model. A theoretical momentum transfer of $1.23 \%$ was calculated in section 3.2 for a deviation angle $\alpha$ of $9^{\circ}$. The images from the experiments however showed that the gelatine birds did not deviate over an angle of $9^{\circ}$, but rather $5-6^{\circ}$, which corresponds to approximately $0.46 \%$ momentum transfer. Because the actual tip of the splitter is much shorter compared to the plates of the wedge (see also Figure 5 and 6 ), it is possible that the deviation angle is lower than the one defined by the splitter tip. There are two possible explanations for the higher momentum transfer in the experiments : (i) the assumption $v_{b, 2}=v_{b, 1}$ is not correct or (ii) some friction might be at play. The ducks deviate even less $\left(3-4^{\circ}\right)$, which corresponds to a negligible momentum transfer as a result of a change in momentum direction, while the momentum transfer is almost double the one from gelatine. The fact that the ducks deviate considerably less than the gelatine birds is an interesting observation. This indicates that gelatine is capable of storing (much more) elastic energy.

The higher momentum transfer of the real birds compared to the gelatine can also be observed in the momentum transfer over time curves shown in Figure 19. Contrary to the wedge, these curves are derived from integrated accelerometer signals. From the three considered targets in this paper, the splitter is the most rigid, the accelerometer is attached firmly to the structure and can therefore be used to obtain very accurate momentum over time signals. The connection failed for one of the 1:6 MR experiments. For the two 1:4 MR birds, drift was present on the acceleration signal. The drift is subtracted from the original signal, which results in quite comparable graphs for the first slope.

For the three experiments with gelatine birds, the slope and therefore force is quite similar. For the two experiments with ducks, the slope or force is also consistent, but considerably higher. The final momentum transfer is almost three times higher for the real birds. The substitute birds clearly underestimate the force and momentum transfer of the real birds, contrary to the plate and wedge experiments. The energy balance is very important in this experiment. For the gelatine birds for example, the kinetic energy of a 3 gram piece of bird is so large that it would double the final speed of the splitter when that piece of bird is decelerated from the initial bird velocity to the velocity of the splitter. On the high speed images, pieces of duck sticking to the splitter due to tendon, feathers and so on can be observed (images are not shown) which can increase the energy transfer by a large extent. For the gelatine experiments, this is not observed. The mass that sticks to the splitter could be the reason for the higher force, but the question remains if this is the only mechanism that generates force.

Disregarding the sticking of tendon and feathers, the slopes are quite constant for the real birds, which gives an indication that the bird, including bones, feathers, flesh and so on, interacts quite homogeneously at these impact speeds. 


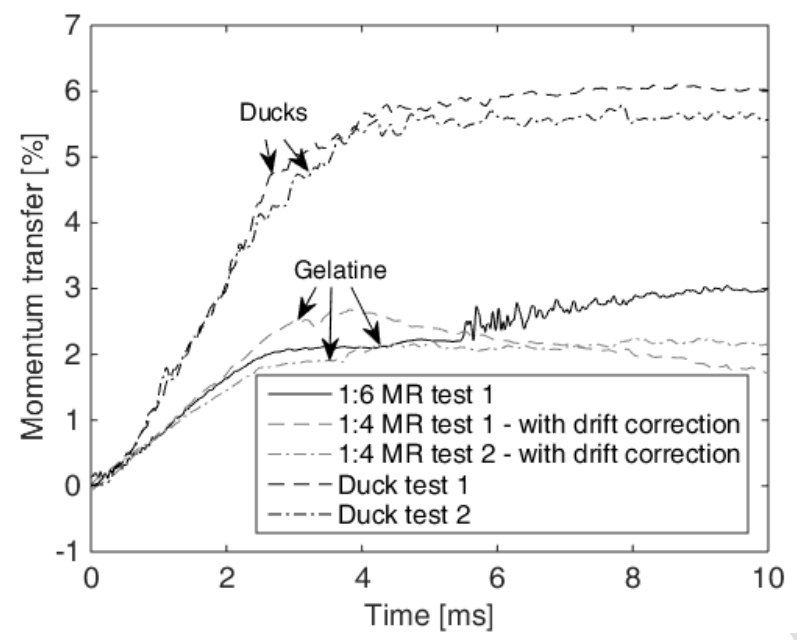

Figure 19: Momentum transfer curves for several splitter experiments, obtained from the accelerometer signals.

In general, the forces induced on the splitter are much lower compared to the wedge and the plate. For each of the six experiments, the force is lower than $2 \mathrm{kN}$. Compared to the 50-100 kN seen in the plate experiments (for the same range of initial momentum), splitting forces (as far as it is actually about splitting), should have a minor effect in an impact event with a considerable change of momentum direction. The difference between the ducks and the gelatine is relatively smaller for the momentum transfer (Figure 18a) compared to the force (Figure 18b), because the ducks are longer and therefore the impact time is a bit higher compared to the gelatine birds.

In Figure 20, a 1.5kg 1:4 MR gelatine bird impact at $106 \mathrm{~m} / \mathrm{s}$ on the splitter target is shown.

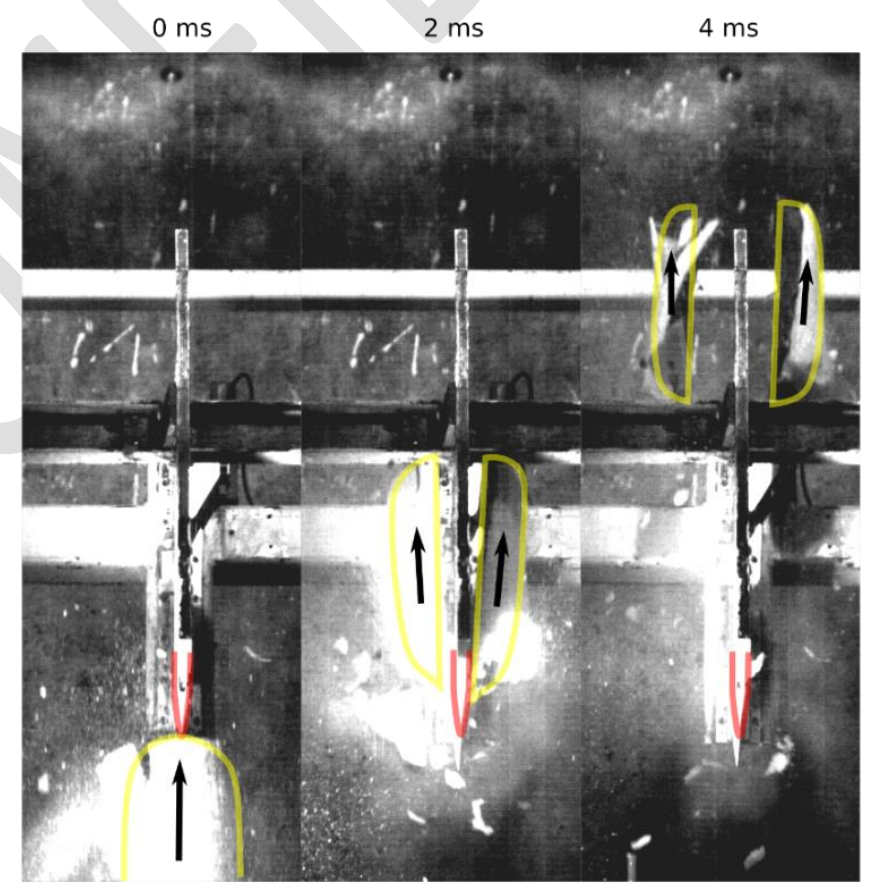

Figure 20: Impact of $1.5 \mathrm{~kg}$ 1:4 MR gelatine bird (yellow contour) on the splitter (red contour). 
The figure shows that the gelatine bird remains in two pieces after impact. There are no pieces of gelatine that stick to the splitter.

\subsubsection{Simulations}

Several splitter simulations are performed with the same input parameters as the experiment with the 1:6 MR gelatine bird with an impact momentum of $135 \mathrm{Ns}$ (Figure 18). Figure 21 shows the final momentum transfer for five mesh sizes, including the theoretically determined $1.23 \%$. The influence of the mesh size is relatively higher for the splitter simulations. The momentum transfer seems to decrease linearly with decreasing mesh size. For a decreasing mesh size, the elastic energy also keeps decreasing. As a result, the meshes also deviate less for a decreasing mesh size, as shown on the right in Figure 21 for two mesh sizes. The upper part only shows the contour of the $2.52 \mathrm{~mm}$ mesh in green which deviates more from the impact direction (the dashed line) than the $1.59 \mathrm{~mm}$ mesh in blue. The absence of deviatoric and/or dissipating constitutive behaviour might again be the reason that the momentum transfer is slightly underestimated for the finer meshes, although the momentum transfer is still very well in line with that of the gelatine birds in Figure 18a and Figure 19.
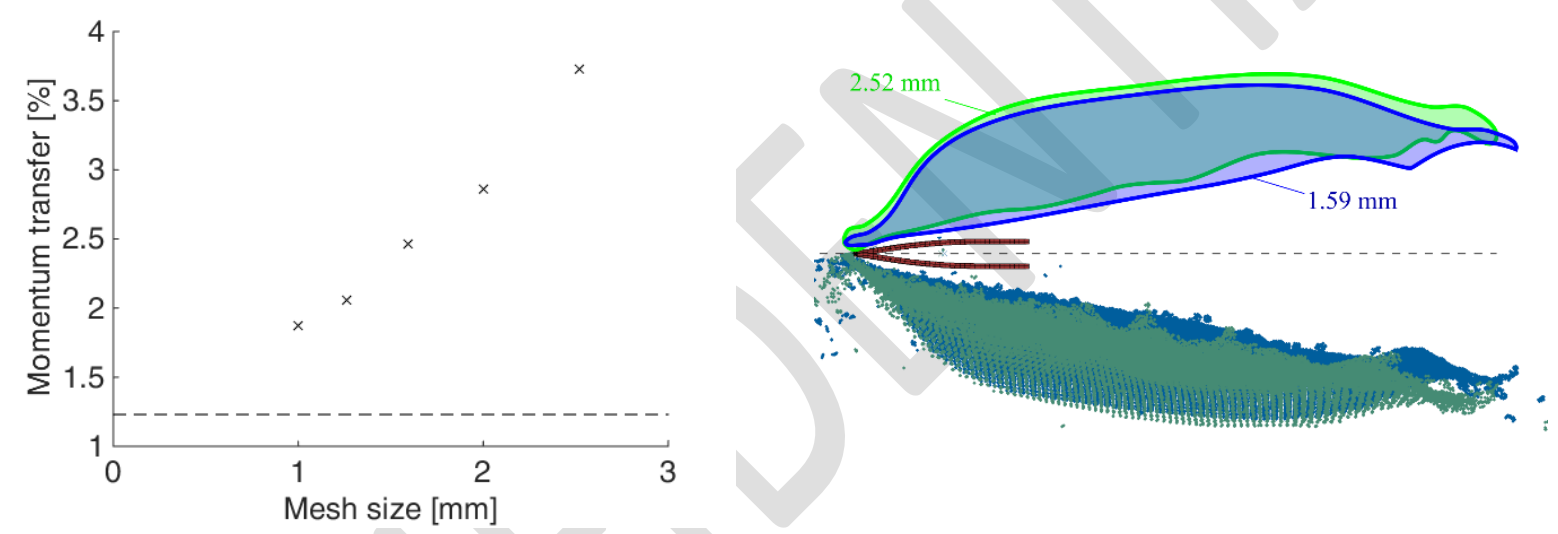

Figure 21: Final splitter momentum transfer in function of the mesh size and a comparison of the deviation of two different mesh sizes.

\section{6. $\quad$ Post-impact residual energy measurement}

Within the context of the European project E-Break, a set-up to measure the residual energy of the bird remainders after a first impact was of high importance. Several attempts to make a "catcher box" which could catch the bird remainders and therefore measure its kinetic energy were unsuccessful. The experimental momentum transfer results of the plate structure as well as numerical verification of an impact with defragmented bird pieces however prove that the residual energy can be simply measured with the rigid plate structure.

The plate experiments and simulations learned that $100 \%$ (101.2\% in the experiments) of the impact momentum is transferred to the plate, independent of the shape, mass or velocity. This fact can be used the other way around, instead of determining the momentum transfer from the impact momentum of the bird and the momentum after impact of the target, the momentum of the bird can be determined from the momentum of the target and the assumption that $100 \%$ of the momentum is transferred. The obtained momentum of the bird in the direction orthogonal to the plate can be used to obtain the velocity of the bird, from which the residual energy in the direction of impact can be calculated. 
The concept is validated numerically. Figure 22 shows the simulation of a secondary impact of a deformed bird on a rigid plate (the bird first hits another object which is not visible on the figure) together with the momentum transferred to that plate. An additional simulation is performed without the plate to obtain a reference impact momentum indicated by the dashed rectangle of the bird orthogonal to the plate or also the x-direction. The momentum transfer is obtained by dividing the momentum of the plate by the reference impact momentum of the deformed bird in the $x$ direction. As can be observed in Figure 22, after impact on the plate, the deflected mass does not exactly move orthogonal to the $x$-direction, there is some momentum in the negative $x$-direction. Obtaining this momentum from the simulation and adding it to the momentum of the plate results in a momentum transfer of $107.5 \%$ instead of $100 \%$. The momentum curve of the plate indeed ends up higher than $100 \%$ and quite close to the $107.5 \%$. In the experiments however returning mass is never observed, so for the mass that hits the plate, a momentum transfer of $100 \%$ can be assumed.
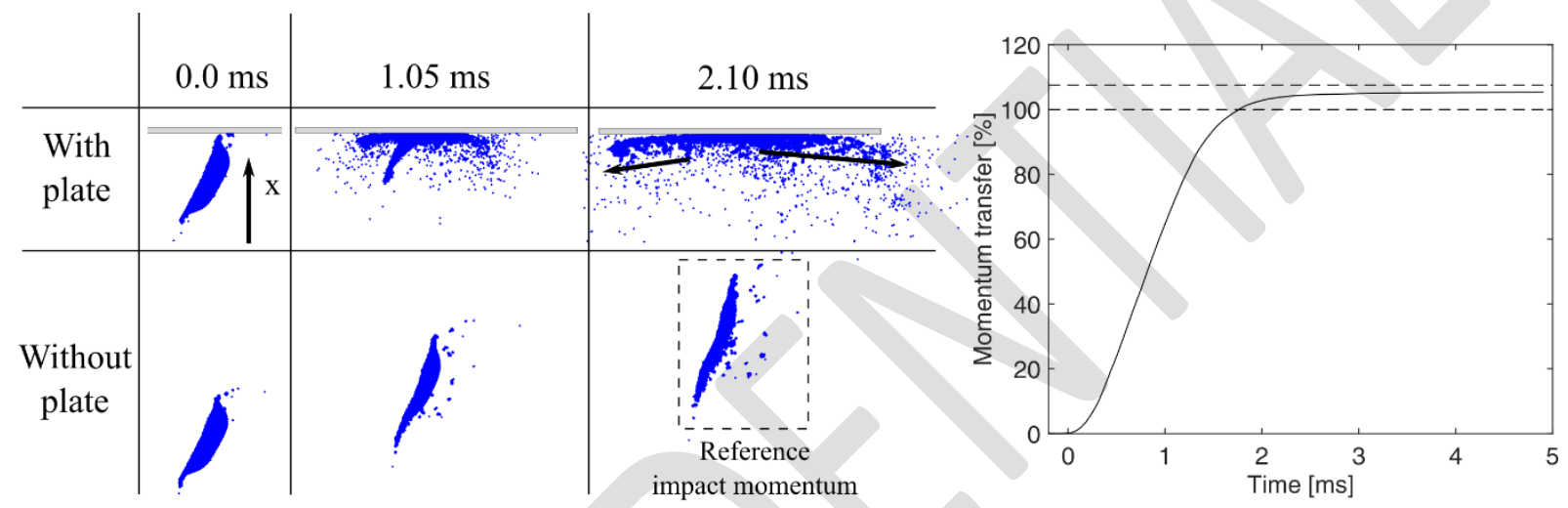

Figure 22: Momentum transfer for a highly deformed bird.

\section{Conclusions}

Bird impact experiments on a rigid plate, wedge and splitter target with gelatine and real birds have been performed and investigated. In this study, the momentum transfer proved to be a good measure to compare birds with different materials, masses, lengths and speeds with each other. To study the impact on these rigid targets, a new principle to acquire the momentum transfer in bird strike experiments has been introduced and validated. The results of the different experiments and simulations can be summarized as follows:

Gelatine versus real birds: Especially for the plate and the wedge, fluid-like impactors have comparable mass flows and therefore comparable average impact forces and momentum transfer. As a result, the impactor material (gelatine or real bird) has a negligible influence in this kind of experiment. Different gelatine mixing ratios (MRs) were tested as well as real birds. A 1:9 MR bird proved to perform too liquid, while a 1:4 and 1:6 MR bird often disintegrated into several big lumps. The main difference between gelatine and real birds can be found for the splitter, where the real bird material is more likely to get stuck on the splitter and increases the momentum transfer (and therefore also force) by a large extent, due to its high kinetic energy.

- The momentum transfer: The impact force is mainly driven by the change of momentum direction, as predicted by the analytical models. The momentum transfer for the plate, wedge and splitter experiments is respectively $100 \%, 50-60 \%$ and $2-6 \%$. Compared to the 
experiments and analytical predictions, similar momentum transfer values are obtained from the numerical model for impacts on a flat or rotated plate. But some deviatoric and/or dissipating constitutive behaviour is missing in the numerical model of the bird to acquire a momentum transfer conform the experiments for the wedge and possibly also for the splitter. The results show that the wedge target can be a very valuable set-up to test bird material models in the future because the momentum transfer depends greatly on the constitutive behaviour of the bird material. The average splitter forces are quite low compared to the plate and wedge. For the splitter simulations, the momentum transfer is very dependent of the mesh size.

- Residual energy measurement: The rigid plate can be used to measure the residual energy of a bird after an impact event because the momentum transfer for fluid-like impactors is $100 \%$, independent from the type of bird, shape, mass and impact velocity. This can be deduced from the performed experiments, simulations and the analytical model.

The performed experiments and simulations prove that rigid targets can still be valuable in bird strike research because it allows to focus on the bird behaviour contrary to the otherwise highly coupled impact event.

\section{$\underline{\text { Acknowledgements }}$}

The authors would like to thank Techspace Aero for the cooperation throughout the project. The research leading to these results has received funding from the European Union's Seventh Framework Programme FP7/2007-2013 under grant agreement $n$ _ ACP2-GA-2012-314366-E-BREAK. More information can be found at http://www.e-break.eu/.

\section{References}

1. Wilbeck, J.S., L. Air Force Materials, and L. Air Force Wright Aeronautical, Impact behavior of low strength projectiles 1978, Wright-Patterson Air Force Base, Ohio: Air Force Materials Laboratory, Air Force Wright Aeronautical Laboratories, Air Force Systems Command.

2. Wilbeck, J.S. and J.L. Rand, The Development of a Substitute Bird Model. Journal of Engineering for Power-Transactions of the Asme, 1981. 103(4): p. 725-730.

3. Barber, J.P., H.R. Taylor, and J.S. Wilbeck, Bird impact forces and pressures on rigid and compliant targets1978, Wright-Patterson Air Force Base, Ohio; Alexandria, Va.: Air Force Flight Dynamics Laboratory, Air Force Systems Command ; Distributed by Defense Technical Information Center, Defense Logistics Agency.

4. Barber, j.P., et al. Characterization of Bird Impacts on a Rigid Plate: Part 1. 1975; Available from: http://handle.dtic.mil/100.2/ADA021142.

5. Challita, A. and j.P. Barber, The Scaling of Bird Impact Loads, 1979, University of Dayton Research Institute: Dayton, Ohio. p. 1-66.

6. Challita, A. and I. Dayton Univ Oh Research. Validation of a Bird Substitute for Development and Qualification of Aircraft Transparenciles. 1980; Available from: http://handle.dtic.mil/100.2/ADA097736.

7. Challita, A. and B.S. West, Effects of Bird Orientation at Impact on Load Profile and Damage Level, 1980, University of Dayton Research Institute: Dayton, Ohio. p. 1-85.

8. Allcock, A.W.R. and D.M. Collin, The Development of a Dummy Bird for Use in Bird Strike Research1969: H.M. Stationery Office.

9. Lavoie, M.A., et al., Bird's substitute tests results and evaluation of available numerical methods. International Journal of Impact Engineering, 2009. 36(10-11): p. 1276-1287. 
10. Roberts, G.D., et al., Ballistic impact of braided composites with a soft projectile. Journal of Aerospace Engineering, 2005. 18(1): p. 3-7.

11. Liu, J., Y.L. Li, and X.S. Gao, Bird strike on a flat plate: Experiments and numerical simulations. International Journal of Impact Engineering, 2014. 70: p. 21-37.

12. Heimbs, S., Bird Strike Simulations on Composite Aircraft Structures, in Simulia Customer Conference2011.

13. Heimbs, S. and T. Bergmann, High-Velocity Impact Behaviour of Prestressed Composite Plates under Bird Strike Loading. International Journal of Aerospace Engineering, 2012.

14. Hou, J.P. and C. Ruiz, Soft body impact on laminated composite materials. Composites Part aApplied Science and Manufacturing, 2007. 38(2): p. 505-515.

15. Premont, E.J. and K.R. Stubenrauch, Impact Resistance of Composite Fan Blades, 1974, Aircraft Engine Group, General Electric Co.: Cincinnati, Ohio. p. 1-143.

16. Ivančević, D. and I. Smojver, Hybrid approach in bird strike damage prediction on aeronautical composite structures. Composite Structures, 2011. 94(1): p. 15-23.

17. Azevedo, R.L. and M. Alves. Numerical Simulation of Bird Strike Impact Against Balanced Fiberglass/Epoxy Composite Plates. in 19th International Congress of Mechanical Engineering. 2007.

18. Bertke, R.S. and J.P. Barber, Impact Damage on Titanium Leading Edges From Small Soft-Body Objects, 1979, University of Dayton Research Institute: Dayton, Ohio.

19. Seidt, J.D., et al., Dynamic Load Measurement of Ballistic Gelatin Impact Using an Instrumented Tube, in XII International Congress and Exposition on Experimental and Applied Mechanics2012.

20. McCarthy, M.A., et al., Modelling of Bird Strike on an Aircraft Wing Leading Edge Made from Fibre Metal Laminates - Part 2: Modelling of Impact with SPH Bird Model. Applied Composite Materials, 2004. 11(5): p. 317-340.

21. Guida, M., Study, design and testing of structural configurations for the bird-strike compliance of aeronautical components, in PhD thesis, U.o. Naples, Editor 2008: Italy.

22. Guida, M., et al., Analysis of Bird Impact on a Composite Tailplane Leading Edge. Applied Composite Materials, 2008. 15(4-6): p. 241-257.

23. Georgiadis, S., et al., Bird-strike simulation for certification of the Boeing 787 composite moveable trailing edge. Composite Structures, 2008. 86(1-3): p. 258-268.

24. Guida, M., et al., Certification by birdstrike analysis on C27J fullscale ribless composite leading edge. International Journal of Impact Engineering, 2013. 54: p. 105-113.

25. Zhu, S., et al., Numerical Simulation of Bird Impact on Fibre Metal Laminates. Polymers \& Polymer Composites, 2014. 22(2): p. 147-156.

26. Liu, J., Y. Li, and X. Gao, Bird strike on a flat plate: Experiments and numerical simulations. International Journal of Impact Engineering, 2014. 70: p. 21-37.

27. Anghileri, M. and G. Sala. Theoretical assessment, numerical simulation and comparison with tests of birdstrike on deformable structures. in Proceedings of the 20th ICAS congress. 1996. Sorrento, Italy.

28. Kermanidis, T., et al., Development and Validation of a Novel Bird Strike Resistant Composite Leading Edge Structure. Applied Composite Materials, 2005. 12(6): p. 327-353.

29. Cronin, D.S., Ballistic Gelatin Characterization and Constitutive Modeling, in Conference on Experimental and Applied Mechanics, T. Proulx, Editor 2011, Springer Science \& Business Media. p. 51-55.

30. Shepherd, C.J., et al., The Dynamic Behaviour of Ballistic Gelatin. AIP Conference Proceedings, 2009. 1195(1): p. 1399-1402.

31. Subhash, G., et al., Non-Newtonian Behavior of Ballistic Gelatin at High Shear Rates. Experimental Mechanics, 2012. 52(6): p. 551-560.

32. Heimbs, S., Computational methods for bird strike simulations: A review. Computers \& Structures, 2011. 89(23-24): p. 2093-2112. 
33. Rosenblatt, M.a.E., G.E. and DeAngelo, L.A. and Kreyenhagen, K.N., Numerical Analyses of Soft Body Impacts on Rigid and Deformable Targets, 1976, California Research and Technology, Inc.

34. Boehman, L.I.a.C., A. , A model for predicting bird and ice impact loads, 1982, University of Dayton Research Institute.

35. Hirschbein, M.S., Bird impact analysis package for turbine engine fan blades, 1982, NASA, Lewis Research Center.

36. Lavoie, M.A.a.G., A. and Nejad Ensan, M. and Zimcik, D.G. , Validation of Available Approaches for Numerical Bird Strike Modeling Tools. International Review of Mechanical Engineering, 2007.

37. Lavoie, M.A.a.G., A. and Ensan, M. Nejad and Zimcik, D.G. , Review of existing numerical methods and validuation procedure available for bird strike modelling. International Conference on Computational \\& Experimental Engineering and Sciences, 2007. 2: p. 111118.

38. Lavoie-Perrier, M.-A., Soft body impact modelling and development of a suitable meshless approach, 2008, Université Laval.

39. Jain, R.a.S., Effect of Bird Material and Projectile Shape on Temporal Pressure during Bird Impact. Proceedings of the Altair's user conference, 2006.

40. Meguid, S.A.a.M., R.H. and Ng, T.Y. , FE analysis of geometry effects of an artificial bird striking an aeroengine fan blade. International Journal of Impact Engineering, 2008. 35(6): p. 487-498.

41. Mao, R.H.a.M., S. A. and Ng, T. Y., Transient three dimensional finite element analysis of a bird striking a fan blade. International Journal of Mechanics and Materials in Design, 2008. 4(1): p. 79-96.

42. Langrand, B.a.B., A.-S. and Chauveau, Y. and Deletombe, E. , Assessment of multi-physics FE methods for bird strike modelling-Application to a metallic riveted airframe. International Journal of Crashworthiness, 2002. 7(4): p. 415-428.

43. Gelatins, P. [cited 20165 May]; Available from: http://www.pbgelatins.com/.

44. EASA Certification Specifications. 2015; Available from: http://easa.europa.eu/documentlibrary/certification-specifications.

45. Liu, G.R. and M.B. Liu, Smoothed particle hydrodynamics : a meshfree particle method2003, New Jersey: World Scientific. $x x, 449$ p. 